\title{
Harmonic Love wave devices for biosensing applications
}

\author{
M.I.Newton, F.Martin, K.Melzak, E.Gizeli and G.MCHale
}

Abstract

We have investigated simultaneous operation of a Love wave biosensor at the fundamental frequency and third harmonic, including the optimization of IDT metalization thickness. Data is presented showing a sequence of deposition and removal of a model mass layer of palmitoyl-oleoyl-sn-glycerophosphocholine (POPC) vesicles whilst frequency hopping between $110 \mathrm{MHz}$ and $330 \mathrm{MHz}$.

Introduction: A Love wave is produced in a surface skimming bulk wave (SSBW) device when an insulating overlayer with an acoustic shear velocity less than that in the bulk is deposited over the propagation path [1]. The overlayer has the effect of confining energy to the surface and hence acting as a guiding layer. The use of Love wave devices for biosensing applications was first reported in 1992 [2] and has since attracted much attention [3-7]. The surface mass sensitivity of acoustic wave devices is known to increase with frequency [3]. However, for liquid sensing applications only a thin layer of fluid, called the penetration depth, above the sensor is probed [4]. This penetration depth, $\delta$, reduces as the frequency increases according to $\delta=(2 \eta / \rho \omega)^{0.5}$ where $\omega$ is the angular frequency, $\eta$ is the viscosity and $\rho$ the density of the fluid. When biological materials such as a vesicle layer are deposited, they may initially be attached to the mass sensitive surface as a series of spheres, in our case of diameter 50nm, which then can be opened out to form a monolayer or bilayer. The biological mass layer within the penetration depth thus changes and an 
acoustic signal response is observed [9]. Here we present data showing a Love wave device operated in a frequency hopping mode utilizing both the fundamental and third harmonic during a biosensing experiment.

Experimental: In this work we have used a split-finger (double-double) interdigital transducer (IDT) design which, for Rayleigh wave devices, is known to resonate with equal strength at both the fundamental frequency and third harmonic. Devices were fabricated on ST-cut quartz with propagation orthogonal to the crystalline $\mathrm{X}$ direction, which is known to support a SSBW, and designed for operation at a fundamental frequency of $110 \mathrm{MHz}$. Each IDT was of length $40 \lambda$ and aperture $65 \lambda$ where the wavelength $\lambda=45 \mu \mathrm{m}$; finger widths were $6.75 \mu \mathrm{m}$ and spacings were $4.5 \mu \mathrm{m}$. The path length was $7 \mathrm{~mm}$ and the guiding layer consisted of Novolac photoresist dissolved between 5\% and 20\% (w/v) in 2-ethyoxyethylacetate (2-EEA) and spin coated at $4000 \mathrm{rpm}$ for 40 seconds; after deposition the films were cross linked by baking in an oven at $200^{\circ} \mathrm{C}$ for 2 hours. Prior to the biosensing experiment these layers were made hydrophilic by treatment with a sol-gel derived silica coating. Hydrochloric acid was used to accelerate and polymerize a silica solution. The gel was then placed on the Novolac surface for 1 minute and washed off with deionised water. A flow cell configuration was used for the biosensing experiments. A silicone gasket was used to isolate the SAW path from the IDTs and liquid was passed through the flow cell at a rate of $0.083 \mathrm{ml} / \mathrm{min}$. The insertion loss of the device was continually monitored using a Hewlett-Packard 4195A network analyzer with markers set at the fundamental frequency and third harmonic, effectively 
hopping between each frequency during the experiment. The experimental sequence consisted of initially a buffer solution, phosphate buffered saline (PBS), which was then followed by deposition of $0.2 \mathrm{mg} / \mathrm{ml}$ of vesicles of palmitoyl-oleoyl-sn-glycerophosphocholine (POPC) in PBS. The vesicle layer was then removed by a detergent, t-octyl-phenoxypolyethoxyethanol (Triton) at $0.1(\mathrm{w} / \mathrm{v}) \%$ in PBS followed by another sequence of POPC deposition and removal.

Results and Discussion: To operate a Love wave device at harmonic frequencies, both the IDT metallization thickness and guiding layer thickness must be optimized. In Figure 1 we show the effect of IDT metalisation thickness on the insertion loss at both the fundamental and third harmonic. Previous work [8] has shown that the metallization thickness of the grating is a critical parameter for surface transverse wave (STW) filter operation on this substrate with the third harmonic diminishing with increasing thickness. Our data confirms that is also the case for the IDT thickness for our uncoated SSBW device. All devices used a gold capping layer of $12 \mathrm{~nm}$ with a Ti underlayer varied between $20 \mathrm{~nm}$ to $80 \mathrm{~nm}$; from the data in Fig 1., an IDT metallization thickness of 52nm was chosen for all subsequent experiments. The effect of the Love wave guiding layer thickness was also investigated and the optimum value for observing the third harmonic whilst still retaining a significant fundamental was found to be obtained with $10 \%$ Novolac in 2-EEA. Fig. 2 shows the change in insertion loss as a function of time for a typical biosensing experiment. The mass sensitivity is known to increase with operating frequency and this is shown in a factor of five greater 
change in insertion loss for the harmonic (332.325MHz) than the fundamental (110.925MHz). The rise to the initial insertion loss after the detergent treatment suggests that the POPC is fully removed by the Triton. The greater attenuation obtained during the first POPC deposition is consistent with previously published data [9].

Conclusion: In this work we have demonstrated the possibility of frequency hopping between the fundamental frequency and third harmonic of a Love wave device during the deposition and removal of a biological mass layer. The optimum interdigital transducer metalization and guiding layer thickness for simultaneous operation at these frequencies has been investigated.

Acknowledgment: The authors gratefully acknowledge the financial support of BBSRC provided by research grant 301/E11140.

\section{References}

[1] GULYAEV YV. Review of shear surface acoustic waves in solids. IEEE Trans. Ultrason., Ferroelec., Freq. Control 45 (1998) 935-938

[2] GIZELI E, STEVENSON AC, GODDARD NJ AND LOWE CR. A novel Love-plate acoustic sensor utilizing polymer overlayers. IEEE Trans. Ultrason., Ferroelec., Freq. Control 39 (1992) 657-659

[3] HERMANN F, HAHN D AND BUTTGENBACH S. Separation of density and viscosity influence on liquid-loaded surface acoustic wave devices. Appl. Phys. Let. 74 (1999) 3410-3412 
[4] JACOBY B AND VELLEKOOP MJ. Viscosity sensing using a Love-wave device. Sensors and Actuators A 68 (1998) 275-281

[5] WEISS M, WELSCH W, SCHICKFUS Mv AND HUNKLINGER S.

Viscoelestic behavior of antibody films on shear horizontal acoustic surface wave sensors. Anal. Chem 70 (1998) 2881-2887

[6] JACOBY B AND VELLEKOOP MJ. Analysis and optimization of Love wave liquid sensors. IEEE Trans. Ultrason., Ferroelec., Freq. Control 45 (1998) 1293-1302

[7] HARDING LG and DU J. Design and properties of quartz-based Love wave acoustic sensors incorporating silicon dioxide and PMMA guiding layers. Smart Mater. Struct. 6 (1997) 716-720

[8] BAER RL, AND FLORY CA. Harmonic operation of STW filters. IEEE Ultrasonic symposium (1988) 53-56

[9] GIZELI E, LOWE C.R., LILEY M AND VOGEL H. Detection of supported lipid layers with the acoustic Love waveguide device: Application to biosensors. Sensors and Actuators B 34 (1996) 295-300 
Authors' affiliations:

M.I.Newton, G.McHale and F.Martin (Department of Chemistry and Physics, The Nottingham Trent University, Clifton Lane, Nottingham, NG11 8NS UK) E.Gizeli and K.Melzak (Institute of Biotechnology, University of Cambridge, Tennis Court Road, Cambridge, Cambridgeshire, CB2 1QT. UK)

Corresponding author e-mail: michael.newton@ntu.ac.uk 


\section{Figure Captions}

Fig. 1 Insertion loss for the fundamental frequency (diamonds) and $3^{\text {rd }}$ harmonic (squares) as a function of IDT metalisation thickness.

Fig. 2. Change in insertion loss as a function of time for two POPC depositions and removal using a fundamental frequency of $110.925 \mathrm{MHz}$ (upper line) and third harmonic of $332.325 \mathrm{MHz}$ (lower line). 


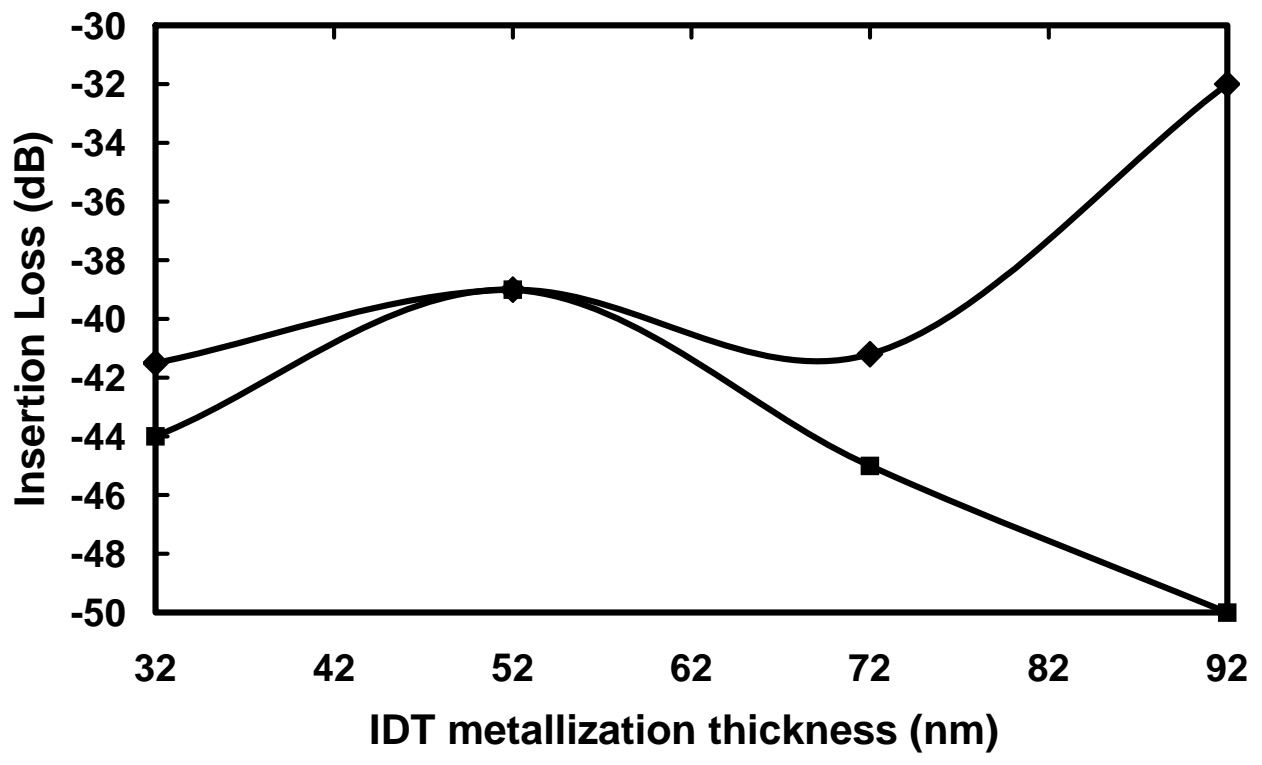




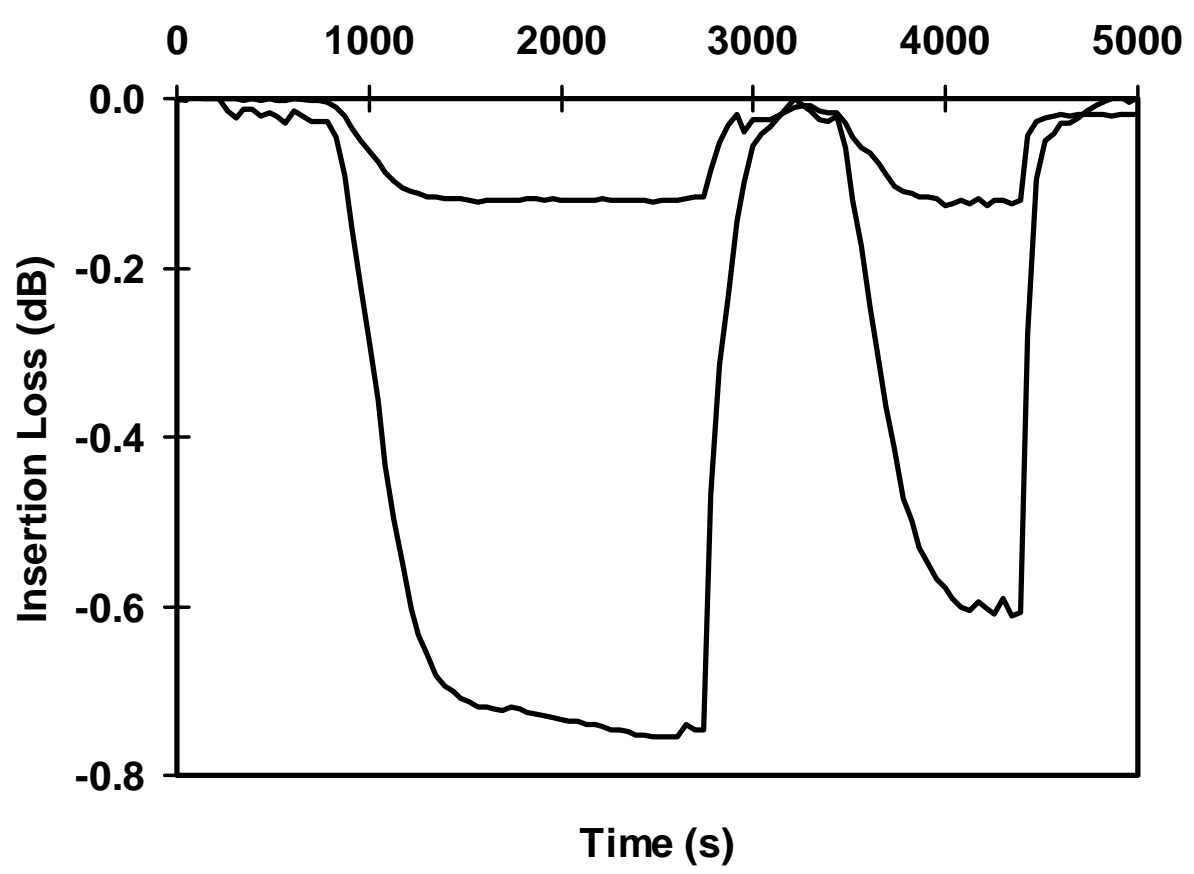

